\title{
Pathogenomics of non-pathogens
}

\author{
Matthew Holden, Lisa Crossman, Ana Cerdeño-Tárraga and Julian Parkhill
}

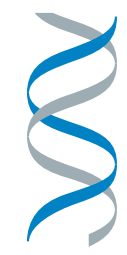

\begin{abstract}
Analysing the genomes of non-pathogenic microorganisms, in addition to its basic and applied scientific interest, can also shed considerable light on the study of pathogenic microorganisms. Two of the three microorganisms described here are rarely pathogenic, but carry genetic determinants that have previously been identified as being important for the pathogenicity of other microorganisms. This underlines the growing understanding that many so-called 'virulence genes' are probably involved in more general interactions between the microorganism and the host or the environment.
\end{abstract}

Staphylococcus epidermidis is generally a relatively benign resident of human skin and mucosal surfaces. However, over recent decades, the increased use of invasive devices and implants, such as catheters, has contributed to the rise of this bacterium as a hospital-acquired pathogen. The severity and diversity of the infections caused by this pathogen are not as great as those associated with its more notorious cousin Staphylococcus aureus, but its ability to form biofilms on artificial surfaces means that it is often recalcitrant to antimicrobial therapy and therefore difficult to treat.

S. epidermidis strain ATCC 12228 is a nonbiofilm-forming, non-infection-associated isolate that has previously been used for antibiotic detection in food products. In their publication, Zhang et al. ${ }^{1}$ present the complete genome sequence, and use previously published genome sequences of clinical S. aureus isolates ${ }^{2,3}$ to identify components of the ATCC 12228 genome involved in virulence, drug resistance and regulation. The authors found that the ATCC 12228 genome contains fewer virulence determinants than the clinical S. aureus isolates - it lacks orthologues of pyrogenic toxins with superantigen activity, and the only toxins identified were the membrane-active $\beta$ - and $\delta$-haemolysins. This strain also lacks the virulence-associated extracellular enzymes staphylokinase, staphylocoagulase and hyaluronidase. It is better equipped with genes encoding adhesins: in addition to previously characterized S. epidermidis adhesin genes (altE, fbe and $e m b P$ ), the genome contains orthologues of several S. aureus MSCRAMMs that have been shown to modulate host-cell interactions. Although the ATCC 12228 isolate therefore appears to be well endowed with potential adhesins capable of binding specific host molecules, it does not contain the ica operon, which is required for polysaccharide-mediated adhesion and biofilm formation in some strains of S. epidermidis. The region of the ATCC 12228 chromosome that is similar to the flanking regions of the ica operon appears to contain signs of genetic rearrangements, leading the authors to suggest that the absence of this operon is the result of a genetic defect.

The comparison of this genome with those of the clinical S. aureus isolates reveals that this strain's virulence arsenal is not as well stocked. However, as this strain is a non-clinical isolate with low virulence in an animal model, the real significance of the arsenal's remaining contents in virulence and colonization is unclear. The apparent loss of the ica operon also raises the question as to whether any virulence functions, or indeed additional biofilm genes, could also be missing. To this end, the publication of the complete genome of the biofilm-forming, clinically isolated S. epidermidis strain RP62A is keenly awaited.

The genome sequence of Chromobacterium violaceum ATCC 12472 reveals a $4.75 \mathrm{Mb}$ single chromosome with $65 \% \mathrm{G}+\mathrm{C}$ content ${ }^{4}$. This unusual free-living microorganism was isolated from a tropical soil and water environment. Approximately one-third of the predicted genes encode transport proteins, which are thought to be highly specific and permit efficient scavenging of very low concentrations of nutrients. C. violaceum can adapt quickly to harsh environmental stress using alternative metabolic pathways for growth depending on the nutrients available. Tight transcriptional control is achieved by many regulators, including several sigma factors. The organism produces violacein - a purple pigment with antimicrobial properties against major pathogens such as Mycobacterium tuberculosis - and its production is under quorum-sensing control. The considerable biotechnological potential of C. violaceum has been explored with its use in methods to synthesize polyhydroxyalkanoates (PHAs), hydrolyze plastic films and solubilize gold without mercury. C. violaceum occasionally infects humans but the genome has no obvious pathogenicity islands; it is suggested that these could be isolate-specific. However, there is an almost-complete type III secretion system and some genes similar to those found in Salmonella typhimurium invasion loci. Insecticidal, nematocidal and antibiotic synthesis genes are also present, giving some indication of the diversity of the environment inhabited by this organism.

Finally, Gloeobacter violaceus PCC 7421 is a unicellular cyanobacterium whose genome consists of a single chromosome of just over $4.5 \mathrm{Mb}^{5}$. Key differences have been found in its genome that set this bacterium apart from the rest of the cyanobacterial group it belongs to; this reflects a considerable phylogenetic distance as the Gloeobacter lineage was the earliest to diverge within the radiation of cyanobacteria and chloroplasts. One of the main differences reflects an ancestral characteristic: G. violaceus has the necessary genes for photosystems I and II, which are both required for oxygenic photosynthesis ${ }^{6}$, however this process occurs in the cytoplasmic membrane and not in the thylakoid membrane, as thylakoids are not present in G. violaceus. Therefore, although the photosynthetic structures present in other cyanobacteria face the lumen of the thylakoids, in this bacterium they face the periplasmic space and hence co-exist with the respiratory system. The other main difference is related to circadian rhythm. G. violaceus lacks the major genetic elements of the circadian clock, the kai genes, which are involved in controlling cell division in other cyanobacteria, suggesting that this system could have been acquired after the divergence of the Gloeobacter lineage.

Matthew Holden, Lisa Crossman, Ana CerdeñoTárraga and Julian Parkhill are at the Sanger Institute, Wellcome Trust Genome Campus, Hinxton, Cambridge CB10 1SA, UK.

e-mail: microbes@sanger.ac.uk

doi:10.1038/nrmicro825

1. Zhang, Y. Q. et al. Genome-based analysis of virulence genes in a non-biofilm-forming Staphylococcus epidermidis strain (ATCC 12228). Mol. Microbiol. 49, 1577-1593 (2003).

2. Baba, T. et al. Genome and virulence determinants of high virulence community-acquired MRSA. Lancet $\mathbf{3 5 9}$, 1819-1827 (2002).

3. Kuroda, M. et al. Whole genome sequencing of methicillinresistant Staphylococcus aureus. Lancet 357, 1225-1240 (2001).

4. BNGP Consortium. The complete genome sequence of Chromobacterium violaceum reveals remarkable and exploitable bacterial adaptability. Proc. Natl Acad. Sci. USA 100, 11660-11665 (2003)

5. Nakamura, Y. et al. Complete genome structure of Gloeobacter violaceus PCC 7421, a cyanobacterium that lacks thylakoids. DNA Res. 10, 137-145 (2003).

6. Hervas, M., Navarro, J. A. \& De La Rosa, M. A. Electron transfer between membrane complexes and soluble proteins in photosynthesis. Acc. Chem. Res. 36, 798-805 (2003). 


\section{O N L I N E}

\section{Online links}

Entrez

S. epidermidis strain ATCC 12228

http://www.ncbi.nlm.nih.gov/genomes/framik.cgi?db=genome\&g

$\mathrm{i}=271$

Chromobacterium violaceum ATCC 12472

http://www.ncbi.nlm.nih.gov/genomes/framik.cgi?db=genome\&g

$\mathrm{i}=321$

Gloeobacter violaceus PCC 7421

http://www.ncbi.nlm.nih.gov/genomes/framik.cgi?db=genome\&g

$\mathrm{i}=346$ 